\title{
Automatic Nipple Detection on Mammograms
}

\author{
Styliani Petroudi and Michael Brady \\ Medical Vision Laboratory, Engineering Science, Oxford University, \\ Ewert House, Ewert Place, Summertown,Oxford, OX2 7BZ, United Kingdom \\ \{styliani, jmb\}@robots.ox.ac.uk
}

\begin{abstract}
We propose an algorithm for automatic, accurate detection of the nipple location on digitized mammograms. The nipple is the principal anatomical landmark on the breast. The proposed algorithm segments the "fat-band" present in the breast edge. The location of the nipple is detected from the curvature of the outline of this band.
\end{abstract}

\section{Introduction}

Reliable diagnosis of abnormalities from a single mammogram is difficult and it is often the case that a mammogram of a woman is compared with previous mammograms, as a basis for evaluation of abnormality. The location of the nipple is invaluable in registration, imaging modality fusion, and as a reference point for classifying microcalcification clusters (milk ducts tend to be oriented approximately towards the nipple). Few methods for nipple detection have been reported and most of them ignore the fact that the nipple may or may not be in profile. In a number of studies the location of the nipple is set to be the point that is furthest away from the pectoralis. All reported methods assume that the skin-air boundary has been correctly identified and that the nipple outline is preserved. Due to the high optical density of the skin and subcutaneous tissue in the breast edge (the "fat-band"), the overwhelming majority of the skin-air segmentation algorithms report problems in preserving the nipple contour. We propose a novel, automatic method to detect the nipple -irrespective of whether it is in profile or not- based on identification of the breast edge and reliable preservation of the location of the nipple. The novel method is based on intrinsic image and anatomy properties of the breast edge border.

\section{Proposed Method}

The new algorithm is the result of observations of the behavior of intensities in the region adjacent to the skin-air interface. During mammogram acquisition, the breast is compressed between two plates; therefore, the breast edge is composed primarily of fat which appears opaque. We refer to this area as the "fat-band". The pixels here have lower mean local intensity and contrast when compared to the rest of the breast and higher mean local intensity and contrast when compared to the background. Local enhancement is achieved by comparing the 
local mean (brightness) and standard deviation (contrast) in the neighborhood of every pixel to the global mean and standard deviation, and adjusting the intensity of the central pixel based on the result (Figure 1b). A binary mask of the "fat-band" may then be assembled by adaptive thresholding (Figure 14). The skin-air boundary is the outer side of the "fat-band". We have observed that the nipple is clearly visible either in the outer or inner side of the "fatband", depending on whether or not it is in profile. We propose the application of a multi-scale approximation of the gradient along the inner and outer sides of the "fat-band". Near the outline of the nipple, the curvature of the boundary increases dramatically and there is a succession of significant curvature changes. From anatomy, the search region for the nipple location may be confined within $+/-3 \mathrm{~cm}$ from the outmost coordinate of the boundary with respect to the side where the pectoral muscle should anatomically be. We evaluate the position of the nipple by the curvature, as the rate of change of the gradient angle. Based on the distance between extreme curvature changes in the outer side of the fat band and the inner side of the fat band we are able to determine whether the nipple is in profile or not and estimate its position.
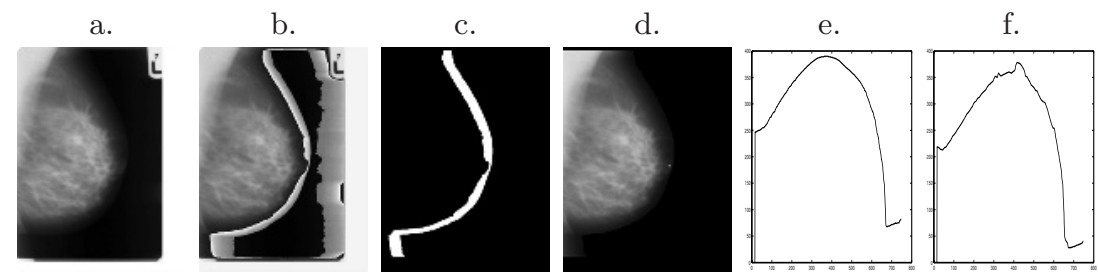

Fig. 1. Applying the new nipple location detection algorithm. (a)The breast mammogram. (b)The breast after local enhancement filtering. (c)The breast "fat-band". (d)The detected nipple position on the segmented breast region. (e)The outside outline of the breast region. (f)The inside outline of the breast region.

\section{Preliminary Results and Discussion}

The algorithm was applied to 20 mammograms subsampled to a resolution of $300 \mu \mathrm{m}$ with a size of $591 \times 802$. The estimated nipple position overlapped a manual nipple outline in all cases and successfully distinguishes whether the nipple is in profile or not. The success of the method must be attributed to exploitation of intrinsic properties of the breast-edge border and the geometry of the nipple. However, more investigation is required for the multi-scale estimation of the gradient and the possible use of monotony operators on the fat-band as a measure of the curvature and for the detection of horizontal structures on the boundary. Since the nipple is the dominant landmark in the breast, correct estimation of its location is important in data fusion, classification of microcalcification clusters, and in 3-D reconstruction of the breast. 